\title{
Research Article \\ Using Nonlinear Diffusion Model to Identify Music Signals
}

\author{
Qiang Li \\ Modern Music School, Shandong University of Arts, Jinan City 250014, China \\ Correspondence should be addressed to Qiang Li; liqiang_xy@sdca.edu.cn
}

Received 7 September 2021; Accepted 27 September 2021; Published 13 October 2021

Academic Editor: Miaochao Chen

Copyright (c) 2021 Qiang Li. This is an open access article distributed under the Creative Commons Attribution License, which permits unrestricted use, distribution, and reproduction in any medium, provided the original work is properly cited.

\begin{abstract}
In this paper, combined with the partial differential equation music signal smoothing model, a new music signal recognition model is proposed. Experimental results show that this model has the advantages of the above two models at the same time, which can remove noise and enhance music signals. This paper also studies the music signal recognition method based on the nonlinear diffusion model. By distinguishing the flat area and the boundary area of the music signal, a new diffusion coefficient equation is obtained by combining these two methods, and the corresponding partial differential equation is discretized by the finite difference method with numerical solution. The application of partial differential equations in music signal processing is a relatively new topic. Because it can accurately model the music signal, it solves many complicated problems in music signal processing. Then, we use the group shift Fourier transform (GSFT) to transform this partial differential equation into a linear homogeneous differential equation system, and then use the series to obtain the solution of the linear homogeneous differential equation system, and finally use the group shift inverse Fourier transform to obtain the noise frequency modulation timedependent solution of the probability density function of the interference signal. This paper attempts to use the mathematical method of stochastic differentiation to solve the key problem of the time-dependent solution of the probability density function of noise interference signals and to study the application of random differentiation theory in radar interference signal processing and music signal processing. At the end of the thesis, the application of stochastic differentiation in the filtering processing of music signals is tried. According to the inherent self-similarity of the music signal system and the completeness and stability of the empirical mode decomposition (EMD) algorithm, a new kind of EMD music using stochastic differentiation is proposed for signal filtering algorithm. This improved anisotropic diffusion method can maintain and enhance the boundary while smoothing the music signal. The filtering results of the actual music signal show that the algorithm is effective.
\end{abstract}

\section{Introduction}

Music signal restoration and enhancement is an important part of music signal processing, and it is a problem of early auditory music signal processing. Music signal preprocessing is the necessary work in the early stage of music signal analysis and processing, such as filtering to reduce the noise of the music signal and enhance the edge of the music signal [1]. Music signal recognition technology plays an important role in improving the quality of music signals and is conducive to the postprocessing of music signals, such as music signal segmentation and music signal tracking [2]. Due to the difficulty in solving the time-dependent solution of the probability density function of the noise interference signal, most of the current researches are based on statistical models, and only the solution method of its steady-state solution is given. The main reason is that the probability density function satisfies that it is difficult to solve the partial differential equations [3]. Traditional linear filtering methods such as median filtering and Wiener filtering can also blur the edges of the music signal while removing noise. When the partial differential equation model is used to restore the music signal, it can remove the noise and keep the edge of the music signal. On this basis, this article uses the impact filter model to improve it, which can make the processed music signal have more edges while removing noise, so that it has the characteristics of music signal recognition [4].

For statistical filters in music signal restoration, for example, median and average filters use the median and 
arithmetic mean values of peripheral pixels in a window to replace the central pixel value. The mean filter is commonly used to filter out Gaussian noise, and the median filter is commonly used to filter out impulse noise [5]. Filters based on partial differential equations can use a broadly continuous two-dimensional function to model the music signal, thereby performing operations such as derivation and integration of the music signal, which standardizes the problem of music signal processing and makes the description of the problem form becomes simple, and the nonlinear filter in music signal processing is reunderstood and analyzed [6]. The use of traditional linear filters to restore and enhance the music signal contaminated by noise will blur or even destroy the discontinuous information of the music signal boundary, while the anisotropic diffusion filter based on partial differential equations can denoise and at the same time well keep the music signal [7]. At the same time, the diffusion method based on partial differential equations has some shortcomings. The most important one is the computational cost of the diffusion equation, which is related to the calculation of continuous integrals. These computational inconveniences make the anisotropic diffusion method difficult to apply to most real-time videos [8].

The main research work of this paper is centered on the application of partial differential equations in music signal recovery and music signal recognition. It mainly analyzes traditional music signal analysis methods and studies the application of partial differential equation methods and anisotropic diffusion equations in music signal restoration, as well as their improved algorithms for music signal recognition. Partial differential equation is a relatively sophisticated method of music signal analysis and processing, which has important research and application value and requires in-depth research. The use of partial differential equations for music signal processing is to process the music signal as a whole. For example, it can well keep the edge shape and position unchanged while denoising. On the basis of summarizing the mathematical model of partial differential equation method in music signal processing, combining partial differential equation and wavelet method, this paper proposes a new denoising algorithm suitable for secondorder partial differential equations and high-order partial differential equations. We focus on the simple and practical wavelet threshold denoising method, and aiming at the shortcomings of its threshold denoising, we propose a denoising method based on wavelet transform and Wiener filtering and introduce the modeling and denoising method of partial differential equation image processing. The experiment shows their effectiveness. The organic combination of the two will overcome their shortcomings and get better results. The variational model and the anisotropic diffusion model have a complete theoretical framework, various models, and mature numerical solutions. The introduction of them into the field of music signal processing and computer hearing undoubtedly provides a powerful tool for solving problems in this field. Therefore, the research topic of this article has a certain theoretical background and strong practical value.

\section{Related Work}

The idea of using partial differential equations for music signal processing was really established from Zhang et al. [9]. They introduced the concept of scale space, which expresses a group of music signals on multiple scales at the same time. The contribution to a large extent constitutes the basis of partial differential equations for music signal processing. The multiple scales of the music signal are obtained by Gaussian smoothing, and the scale space of the music signal can also be obtained by using the classical heat conduction equation to evolve the music signal. Chen et al. [10] proposed that the heat conduction equation is not the only equation that can form a scale space and put forward the criteria for forming a scale space. The anisotropic diffusion model proposed by Farrokhmanesh and Hamzeh [11] is the most influential in this field. They proposed to replace the Gaussian diffusion with a selective diffusion that can maintain the edge, which led to many theoretical and practical problems. Under the same framework, Todisco et al. [12] proposed the method of reducing partial differential equations (TV: total variation) proposed by shock filter, which highlights the importance of partial differential equations in music signal processing.

In the fields of music signal processing and computer hearing, there are some partial differential equations based on curves and surfaces of curvature motion. Shahabi and Moghimi [13] developed a level set numerical algorithm. Their idea is to use a higher-dimensional hypersurface level set to represent deformed curves, surfaces, or music signals. This technique not only makes the numerical results more accurate but also solves the topological structure problem that was difficult to deal with before. Partial differential equations can also be used for music signal segmentation. The model proposed by Ando [14] integrates a variety of music signal segmentation algorithms, causing many new problems in theory and practice. Some scholars also have a great influence on music signal segmentation algorithms based on active boundaries. Later, many scholars extended their work with geometric partial differential equations [15-17]. Scholars used stochastic differential equations to study the noise spectral density of switched capacitor circuits, linear and nonlinear time-varying circuits, and stochastic differential equations to study the phase noise model of oscillators [18-20]. Some researchers have used stochastic differential equations to study the noise model in mixers, stochastic differential equations to study the noise problems in radio frequency circuits, and stochastic differential equations to study the noise problems in nonlinear timevarying circuits. This type of research is so extensive because the Brownian motion model can naturally correspond to noise and can easily establish related stochastic differential equations. Partial differential equations can also be used in music signal inpainting. It is synonymous with music signal interpolation. It was originally spread from artists who repaired broken artworks by hand in museums. At present, music signal inpainting technology is used in music signals. There are many applications in processing, auditory analysis, and digital technology, such as music signal restoration, 


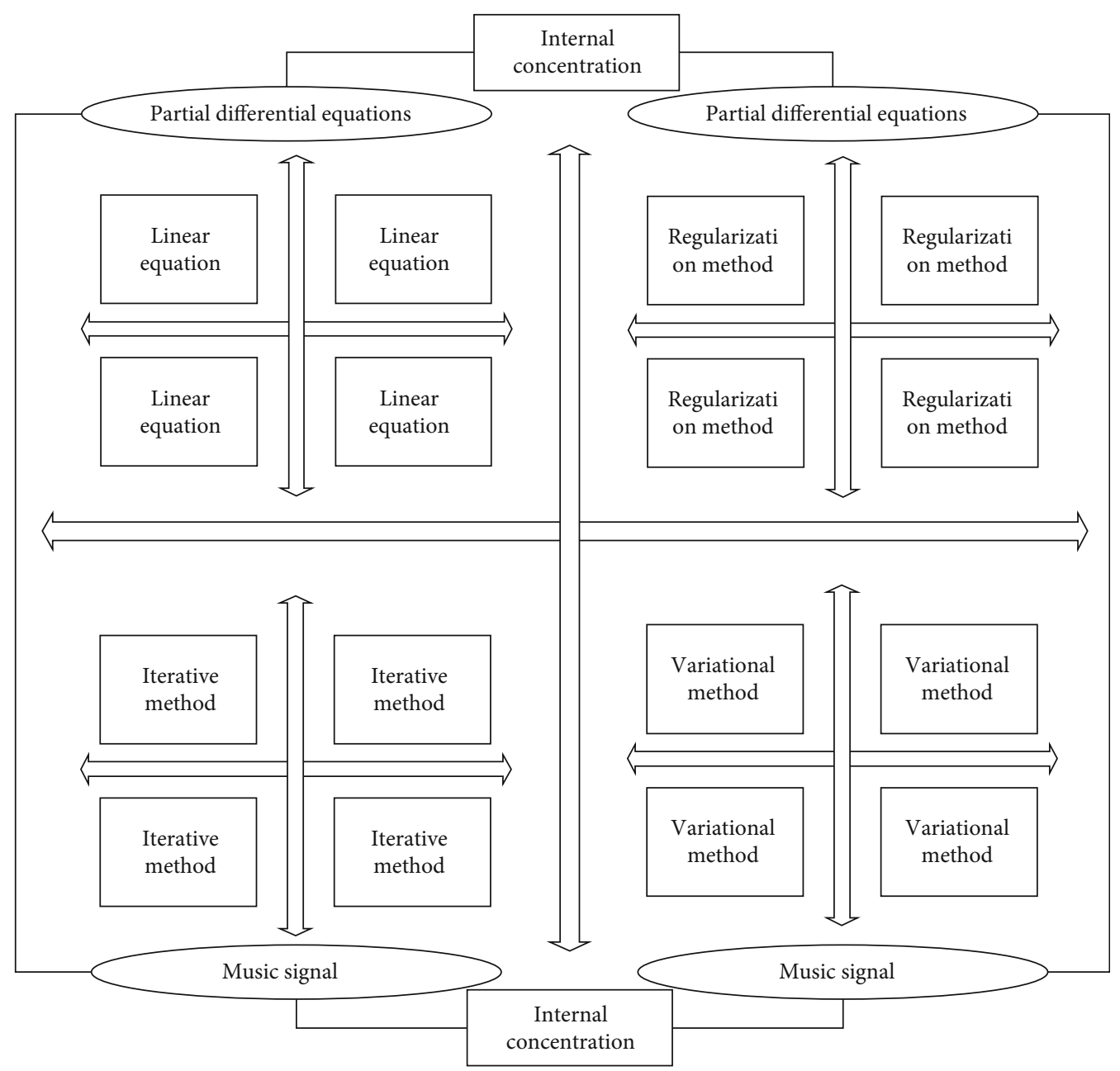

FIgURE 1: The spatial distribution of the solution set of partial differential equations.

music signal amplification, music signal superresolution analysis, and error concealment in wireless music signal transmission [21].

It can be seen that the basic equations used in music signal processing include impact filters, partial differential equations, anisotropic diffusion, and active boundaries, which can realize the recovery, enhancement, and segmentation of music signals. In the smoothing process, the strength and direction of the music signal characteristics are detected at the same time, and the smoothing result is a good combination of noise elimination and feature preservation, which is a better music signal smoothing technology. Compared with the thermal diffusion model, the anisotropic diffusion model is actually a nonlinear parabolic partial differential equation, whose diffusion speed is determined by the gradient of the music signal, which can take into account both noise elimination and feature preservation. The algorithms based on impact filters, partial differential equations, and anisotropic diffusion studied in this paper are important components of many music signal processing methods based on partial differential equations, and they have positive significance for the improvement of these models and numerical calculations.

\section{Construction of a Model for Identifying Music Signals Based on Partial Differential Equations}

3.1. The Solution Set Space of Partial Differential Equations. According to the actual situation of music signal processing, if the problem is transformed into a linear equation system, the spectrum value of the coefficient matrix is generally small, the linear equation system is often weakly conditional, and the solution obtained at this time is often ill-posed. The condition in the theorem is a sufficient condition for solving the problem of definite solution, but it is not necessarily a necessary condition, regardless of whether $A(t)$ satisfies the condition, but as long as it can satisfy the series.

$$
\begin{gathered}
f(x)=\{x(i), i=1, \cdots, n \mid n \in Z\} . \\
\left\{\begin{array}{l}
0<\min p(x)<f(x)-f(x-1) \\
0<(1+a) \times f(x)+f(x-1)<\max p(x) .
\end{array}\right.
\end{gathered}
$$

For this reason, a variety of regularization methods for solving ill-posed problems have been proposed by the 
academic community. These methods focus on maintaining stability while maintaining solution information and use statistical methods, iterative methods, and variational methods to obtain regularized solutions to inverse problems. Figure 1 shows the spatial distribution of the solution set of partial differential equations.

The linear isotropic diffusion filter is the simplest of the music signal smoothing algorithms based on partial differential equations. It has a distinct physical meaning. It can be seen as a process of balancing internal concentration differences when the substance does not produce and die. Its mathematical formula can be expressed as

$$
\begin{gathered}
\lim _{n \longrightarrow \infty}\left(\sum_{i=1}^{n} p(x) \times x(i)-\sum_{j=1}^{n} s(i, j) \times x(j)\right)=R(i, j), \\
g(x)-\sum_{i, j=1}^{n} \frac{\sum_{k=1}^{j} s(k, i)}{s(i, j)}=0 .
\end{gathered}
$$

In music signal processing, the diffusion rate of the linear isotropic diffusion filter is constant; the diffusion rate of the nonlinear isotropic diffusion filter corresponds to the local structure of the music signal; the diffusion rate of the nonlinear anisotropic diffusion filter corresponds to the local structure of the music signal. In the anisotropic diffusion equation, the conductivity at each point is a matrix.

$$
\begin{aligned}
& \sum_{i=1}^{n}|x(i)-f(x)|=n \times f(x), \\
& \sigma(x, y)=\frac{1}{E \times(\varepsilon(x, x)-(1+t)(\varepsilon(y, y))} .
\end{aligned}
$$

Considering that the degradation of the music signal is mainly caused by noise and the noise is regarded as a small target with a large curvature of its contour line, let the isodensity contour line of the noise part with large curvature shrink to a point or even disappear over time, and the music signal part with small curvature evolves into the contour of the music signal and remains.

$$
\begin{gathered}
T=\arg \min \{f(x, t)\}-\arg \min \left\{\frac{\exp (i)}{\exp (t)}\right\}, \\
\{\sigma(x, x), \sigma(y, y), \sigma(z, z)\} \longrightarrow\{\varepsilon(x, y), \varepsilon(y, z), \varepsilon(z, x)\} .
\end{gathered}
$$

Let $y$ be a music signal space, which is defined as a set of piecewise smoothing functionals whose domain is $f$. In this set, only the edges of the steps are discontinuous. The difficulty in extending the energy functional defined in this music signal space is that the gradient amplitude is discontinuous on the edge of the step.

$$
\left\{\begin{array}{l}
\varepsilon(x, x)=\frac{\partial f(x, y, z)}{\partial x}, \\
\varepsilon(y, y)=\frac{\partial f(x, y, z)}{\partial y}, \\
\varepsilon(z, z)=\frac{\partial f(x, y, z)}{\partial z} .
\end{array}\right.
$$

Because the diffusion coefficient is nonzero, it represents a forward degrading diffusion orthogonal to the gradient direction. Since the direction of the edge is perpendicular to the gradient, the well-defined smooth operator that protects the edge trend has received extensive attention.

$$
\begin{gathered}
\int \frac{\partial f(x)}{\partial x} d x=\frac{p^{\prime}(x)}{p(x)}-1, \\
U=\frac{1}{2} \times \int_{\Omega} \sigma(z, x) \sigma(x, y) \sigma(y, z) d \Omega .
\end{gathered}
$$

Usually by setting each element of the thermal conductivity matrix, the diffusion effect is related to the direction of strength. Along the gradient direction, the diffusion effect should be set weaker, especially where the gradient value is large. Along other directions, the diffusion effect should be set stronger, so as to achieve the effect of smoothing and denoising. The design of the thermal conductivity matrix depends on the gradient of the music signal at the current point. Therefore, the diffusion tensor changes with the position of the space.

3.2. Music Signal Recognition Algorithm. The research on the evaluation of the quality of music signal processing is one of the basic researches in the discipline of music signal information. For music signal processing or music signal communication forms, the main body of the information is the music signal, and an important indicator to measure this system is the quality of the music signal. Music signal recognition is to improve the subjective auditory display quality of music signals.

$$
W(x)=\left[\begin{array}{cccc}
\frac{\partial^{2} f(x)}{\partial x^{2}} & 0 & 0 & 0 \\
0 & \frac{\partial^{2} f(x)}{\partial y^{2}} & 0 & 0 \\
0 & 0 & \cdots & 0 \\
0 & 0 & 0 & \frac{\partial^{2} f(x)}{\partial z^{2}}
\end{array}\right]
$$

The principle of music signal restoration is to compensate for the degradation of the music signal so that the restored music signal is as close as possible to the quality of the original music signal. All of these require a reasonable method for evaluating the quality of music signals. 


$$
\begin{gathered}
\sum_{i}|<\alpha(1), u(i)>|^{2}+\sum_{j}|<\alpha(2), u(j)>|^{2}=|u(i, j)|^{2}, \\
\left\{\begin{array}{l}
u(x, y)=\bar{u}(x, y) \\
v(x, y)=\bar{v}(x, y)
\end{array}, x, y \in \bar{S}(u) .\right.
\end{gathered}
$$

The meaning of music signal processing quality includes two aspects: one is the fidelity of the music signal, that is, the degree of deviation between the evaluated music signal and the original standard music signal; the other is the intelligibility of the music signal, which refers to the ability of the music signal to provide information to people or machines.

$$
p(x, t)+\nabla *\left(\frac{\nabla u(x, t)^{k}}{\left|\nabla u(x, t)^{k}\right|}\right)=0 .
$$

The level set method is to regard the music signal as a set composed of equidensity contours, consider that the degradation of the music signal is mainly caused by noise, and regard the noise as mostly small targets with larger curvature of the equidensity contour. So the contour line of the high curvature noise part shrinks to a point or disappears over time, and the low curvature music signal part develops into the contour of the music signal and remains. Figure 2 shows a schematic diagram of the music signal recognition algorithm. Although the most ideal situation is to be able to find out a quantitative description method of the fidelity and intelligibility of the music signal, as a basis for evaluating the music signal and designing the music signal system.

In the real world, music signals captured by photographic equipment are often noisy music signals. For music signals contaminated by noise, it is necessary to denoise the music signals before using traditional music signal recognition algorithms. Then, from the actual situation of music signal processing, when the problem is transformed into a linear equation system, the general spectrum value of the coefficient matrix is small, the linear equation system is often weakly conditional, and the solution is generally ill-posed. The difference between using partial differential equations for music signal processing and traditional methods for music signal processing is that traditional music signal processing methods, such as filters, when performing music signal processing, only need to perform operations on the music signal to get the result, but partial differential equations need to iterate repeatedly on the music signal when processing the music signal, until a stable solution is obtained after several iterations. In the iteration, the music signals of each layer constitute a scale space. To this end, various regularization methods for solving ill-posed problems have been proposed. These methods focus on maintaining stability while maintaining solution information and use statistical methods, iterative methods, and intersection methods to obtain regularized solutions to inverse problems. Some models that use partial differentiation can enhance the edges of the image while removing the noise of the music signal, and the processing speed is fast and the processing accuracy is high.
3.3. Model Weight Factor Replacement. We use the function to represent the music signal, where the music signal domain $Q$ is often taken as a rectangle (2-dimensional), and the function value sweet $(x)$ represents the grayscale (or brightness) of the music signal at the image point $x$. In the computer, the music signal function $u(x)$ processed in the process is a discretized music signal. The points (saraple) are discretely sampled in the music signal domain $Q$. It can be seen that the discovery probability information entropy $H$ begins to decrease with the increase of noise variance $D$, and when it reaches the bottom, it increases with the increase of noise variance $D$. The gray value on each pixel is also discrete, often divided into 128 or 256 levels. For the convenience of theoretical discussion, it may be assumed that the music signal function $u(x)$ is a continuous music signal, that is, assuming that $x$ takes points continuously in the music signal domain, and the gray value at each point is also continuously taken. In order to be able to effectively process music signals, we first need to know how to understand and represent music signals from a mathematical point of view. The music signal model and its representation method determine the music signal processing model to a large extent.

The forward difference format is used in the time dimension. In order to maintain the peak of the music signal, the forward (or backward) difference can be used to calculate $I$ $-x$ and $I-y$. However, the center difference cannot be used because the signal peaks will be smoothed. Finally, four diagonal gradient estimates are added to make the algorithm more sensitive to edge information. Figure 3 shows the two-dimensional scattered point distribution of the sensitivity of the extreme points of the music signal. Due to the small number of iterations, the overall increase in calculations is not much. We can also see that the derived music signal extreme points in the scale space are no more than those obtained in the music signal, which satisfies the requirement of continuous compression and simplification in the scale space representation causality when the scale parameter increases. In this sense, the Gauss convolution kernel is the only one in the continuous scale space of the music signal; that is, the repaired music signal corresponding to the inner scale of the coarse resolution level can be obtained by the convolution operation of the music signal corresponding to the inner scale of the fine resolution level.

Table 1 shows the noise smoothing parameter distribution of music signals. The improved fourth-order partial differential equation has obvious noise smoothing ability. However, the relatively small edges in the music signal are smoothed out together with the impulse noise, especially the details where the edge length is small but the gray level changes on both sides of the edge are large. In a large smooth area, the noise points spread into blocks with similar gray levels, and the smoothing ability needs to be strengthened. Then, group shift Fourier transform is used to transform this partial differential equation into a homogeneous linear differential equation group. Finally, the time-dependent solution of the probability density function of the noise frequency modulation interference signal after the radar intermediate frequency filter is obtained. On the one hand, 


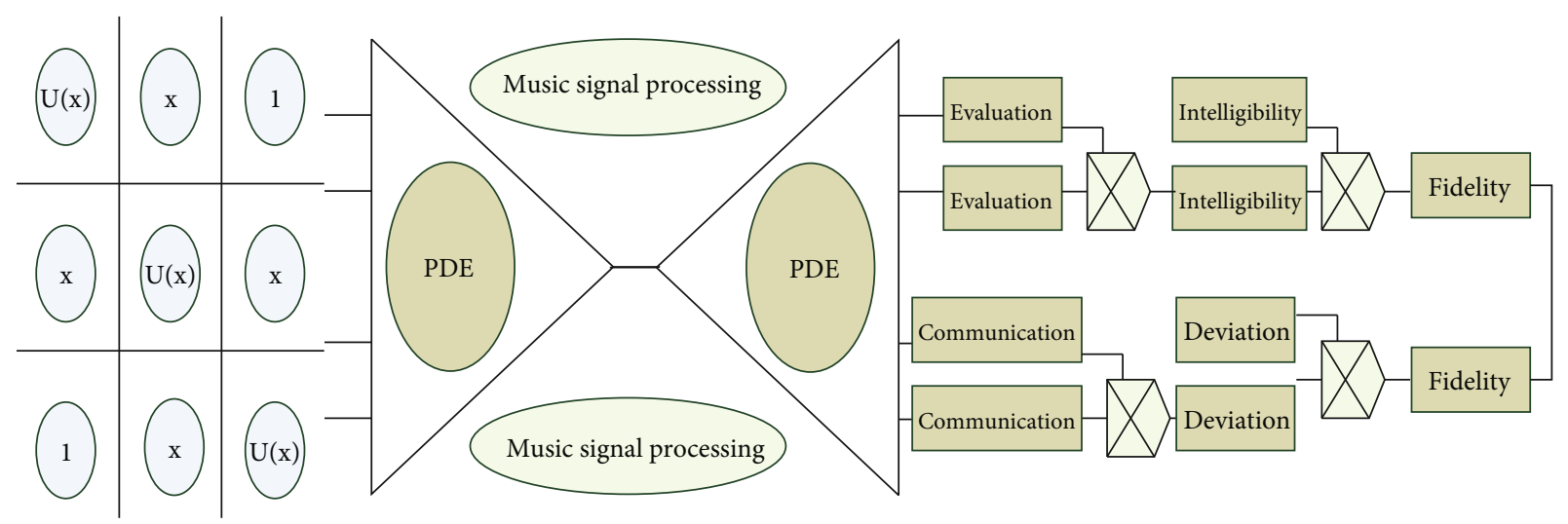

FIGURE 2: Schematic diagram of music signal recognition algorithm.

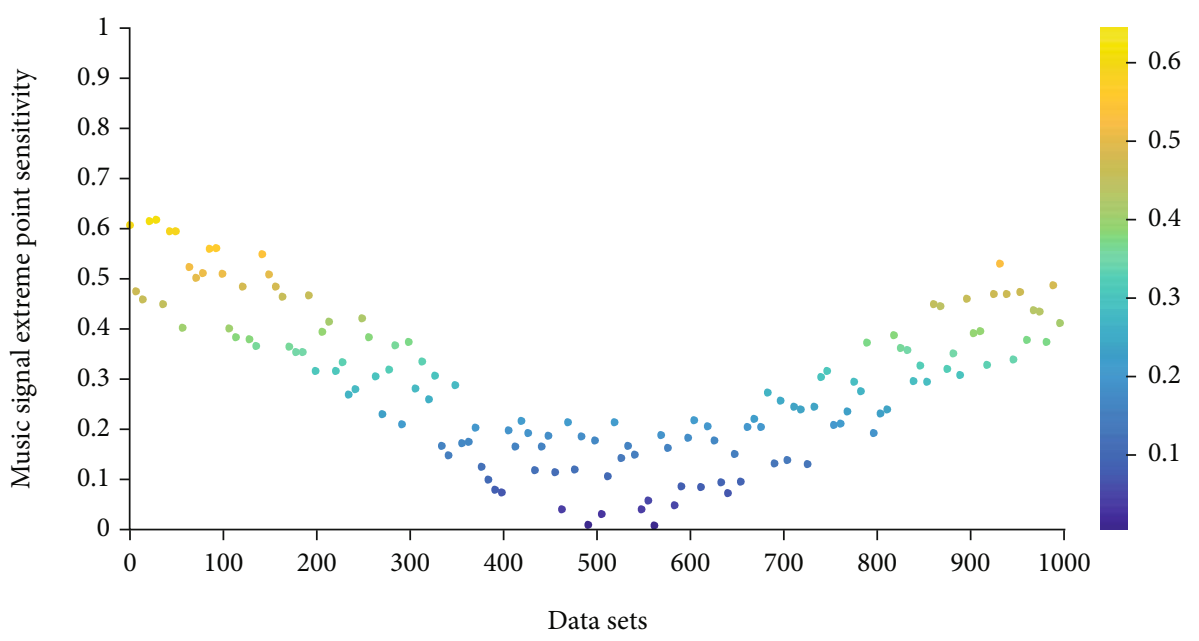

Figure 3: Two-dimensional scattered point distribution of sensitivity of extreme points of music signal.

it performs anisotropic antidiffusion effect on the music signal contaminated by noise; on the other hand, it performs adaptive statistical filtering on the music signal between each antidiffusion, which well removes the obvious pulse points left behind after antidiffusion. We improve the accuracy of the next antidiffusion gradient estimation, greatly reduce the number of iterations of the overall algorithm, and reduce the model's misjudgment of noise points and edge points so that the new algorithm outputs music signals in various areas that are properly smoothed while the edges are still compared sharp and clear.

\section{Application and Analysis of Music Signal Recognition Model Based on Partial Differential Equation Method}

4.1. Music Signal Data Extraction. The input music signal used in the experiment is a music signal with $a=0, b=0.1$ Gaussian noise and $10 \%$ salt and pepper noise, and the size is $404 \times 271$ and $199 \times 199$, respectively. We analyze the output results of various algorithms to compare the performance of the P-M model, forward/backward anisotropic diffusion model, and the proposed partial differential equa-
TABLE 1: Music signal noise smoothing parameter distribution.

\begin{tabular}{lccc}
\hline Parameter index & Signal size $(\mathrm{dB})$ & Smoothing resolution & Weight \\
\hline 1 & 19.71 & 1.32 & 0.13 \\
2 & 20.46 & 1.81 & 0.52 \\
3 & 17.61 & 0.97 & 0.09 \\
4 & 17.13 & 1.37 & 0.26
\end{tabular}

tion algorithm. The objective indicators used for analysis are peak signal-to-noise ratio (PSNR) and mean square error (MSE). The music signals shown in the following are output in the sense of the best PSNR of various algorithms and basically match the output in the sense of the minimum MSE. When the weights of the pixels are the same in each direction, it is the 4-point average smoothing method. It can be seen that partial differential equations can express this isotropic Gaussian smoothing method. In order to ensure the characteristics of the music signal in all directions, it is necessary to improve the partial differential equation into an anisotropic distribution form.

Although the stable range of the parameter is known, in the experiment, an exhaustive method is used to iterate in 
the stable range $(0,0.25)$ with a step size of 0.01 to find the value of that can obtain the best PSNR. Figure 4 shows the music signal peak signal-to-noise ratio matchstick graph. The experimental results show that as long as is selected within the stable range, the best PSNR output will not vary greatly, but the best value is about 0.15 . The numerical results can be seen that compared with the soft stop value method, the method proposed in this paper has a better suppression effect on Gaussian noise in music signals; we can obtain a higher signal-to-noise ratio, effectively reduce noise interference, and better retain the edge part. Information improves the quality of the music signal. A small value will make the algorithm converge more slowly, but it can always converge within 10 iterations. It can be seen that the signal (music signal) is layered using wavelet for a simple hard threshold will cause obvious edge artifacts, which will distort the results. It is worth noting that this method does not increase the amount of calculations. Because the decomposition and reconstruction of wavelets can be completed in a short time using algorithm and the data set in the detail image is sparse, its scale diffusion will be carried out quickly, so the amount of calculations in the whole process will not increase. The partial differential equation is better than the wavelet threshold in this respect, and the processed music signal has a better auditory effect. But the partial differential equation is that the signal is processed as a whole. We can expect the signal (music signal) to be layered and then diffused, which will achieve better results.

4.2. Model Simulation Realization. For two-dimensional grayscale music signals, the effect of median filtering on music signals is usually to take a rectangular window with an odd side length and slide the window along the rows and columns of the music signal point by point. The music signal is at any pixel. The filtered output value is equal to the median value of the gray value of all pixels in the window when the center of the window moves to this point. It is especially important that this method can strengthen the edge and remove noise, while the scale space where the edge is located is more stable than the linear scale space. Obviously, the most important parameter for blurring boundaries in scale space is $K$. If $K$ is too large, the music signal becomes very blurred, which is similar to linear diffusion. If $K$ is small, it can protect the edge well. Therefore, we assume that $K=0$, which is called the stable inverse diffusion equation (SIDES). If each frame of the sequence music signal or video music signal is treated as a separate static music signal, due to this correlation, it can be used to construct the information of several frames of the music signal before and after the music signal to be processed in the continuous video sequence. Based on the anisotropic diffusion model of the sequence music signal to improve the quality of the restored music signal, this section only considers the situation of two frames of images.

The proposed partial differential equation model can output the best music signal in only 4 iterations, and a certain degree of smoothness is obtained in each area, while the edges remain clear and sharp, and there is basically no noise left. Figure 5 shows the histogram of the music signal output recognition rate. The output PSNR has been greatly improved to $21.51 \mathrm{~dB}$, and the MSE is reduced by a minimum of $40 \%$ to 0.356 . It should be noted that comparing the small gray area above each music signal, the output music signal effects of other models almost smooth it out, while the output music signal of the partial differential equation model can be well preserved. Moreover, the edges are more obvious and accurate, and it can be seen that the reproduction of the output music signal of the partial differential equation model is more accurate. With the input of about the size of model, the partial differential equation model requires iterative operations to output the best PSNR music signal generally in about 5 times, and the best PSNR can be improved by about $3 \mathrm{~dB}$ compared with the other algorithms mentioned above.

Figure 6 shows a line graph of the music signal denoising error rate comparison. If the music signal contains a lot of noise, it can be processed by the threshold first, then the appropriate scale diffusion, and finally superimposed to generate the coarse-scale music signal of the original music signal (when the noise is small, the music signal may not be thresholded deal with). It preserves the boundary while removing noise. It is worth noting that this method does not increase the amount of calculation, because the decomposition and reconstruction of wavelet can be completed in a short time by algorithm, and the data set in the detailed music signal is sparse, and its scale is diffused. It will be able to proceed quickly, so the amount of calculations in the entire process will not increase. Wavelet transform uses wavelet coefficients to describe the changes of signals in different scale spaces. If the correct wavelet basis is selected, the signal will be described by some important wavelet coefficients, and at the same time, Gaussian self-noise will contaminate part of the wavelet coefficients. Experiments show that this method can not only improve the efficiency of the algorithm and the signal-to-noise ratio of the music signal but also achieve better auditory effects. It can be seen that the coefficients of each direction are related to the gradient at the point. When the gradient of the point is large (at the edge), the corresponding weight is small, so the degree of smoothness in this direction is small so that the directional feature is retained.

4.3. Example Application and Analysis. First, we perform bicubic interpolation on the low-resolution signal; the interpolation process will bring noise, which is filtered by equations to enhance the graphics while smoothing the noise. The parameters are selected as $c=1, t=0.2$, and $s=0.8$; the iteration step size is 0.02 , iterate 80 times. The experiment successively added Gaussian white noise with a noise variance of 0.02 to the standard image and used the fourth-order partial differential equation method and the method of this article to denoise the image. Among them, the wavelet selects the wavelet to decompose the noisy image in three layers, and $N$ is the total number of pixels of the image. It can be seen from the experimental results that the auditory effect after filtering is more clear. At the same time, it is found through calculation that the signal-to- 


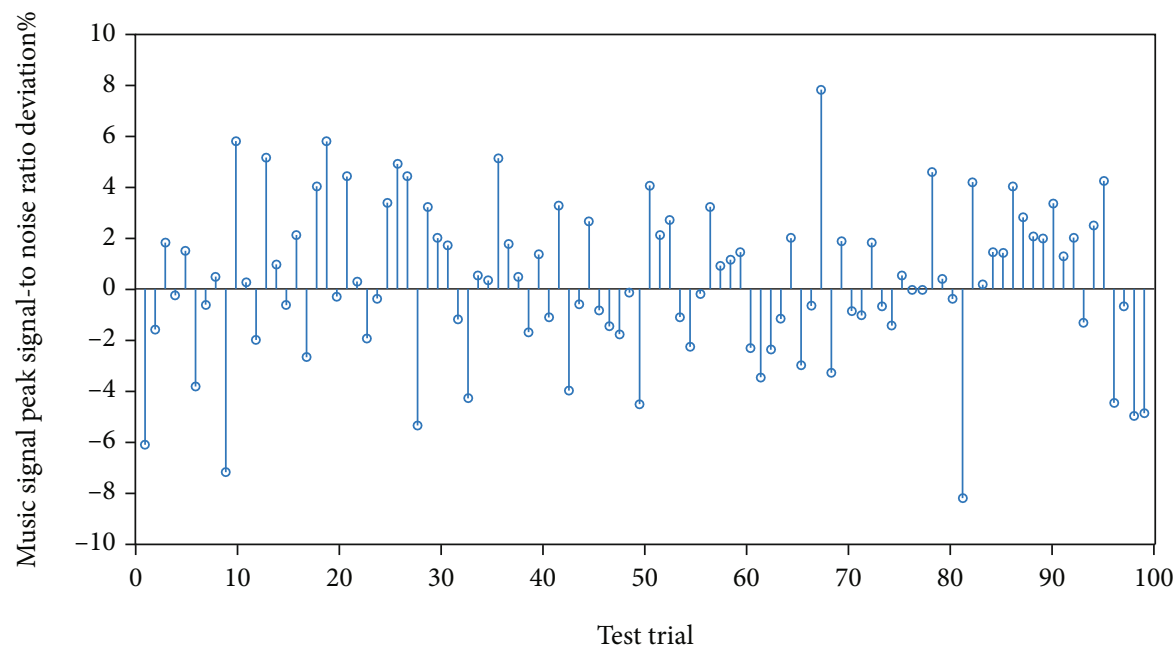

FIGURE 4: Music signal peak signal-to-noise ratio matchstick graph.

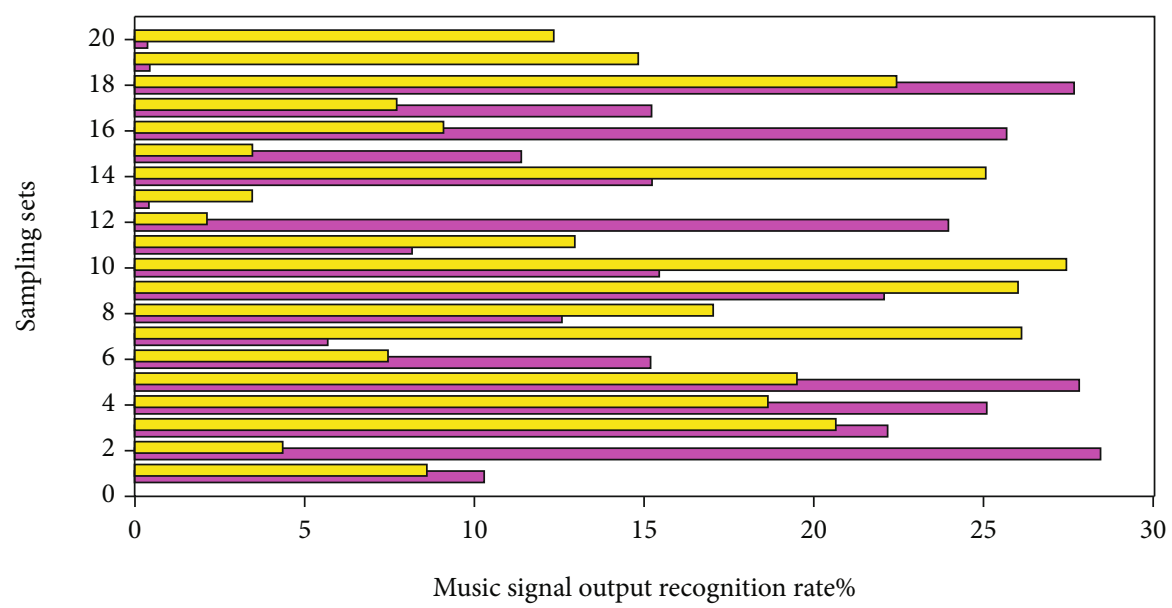

Algorithm1

Algorithm2

FIgURE 5: The histogram of music signal output recognition rate distribution.

noise ratio of the filtered music signal is also improved. It can be seen that the result is obtained after first filtering the music signal with the partial differential equation method and then performing the watershed method for segmentation. The original music signal is filtered, and the time step is $t=0.02$ seconds in the iteration process, and this generation is performed 100 times. It can be seen that when a linear partial differential equation is used to amplify the music signal, no matter how many times it is magnified, the spots and the deviation of bright and dark areas are not obvious, and the effect is better in areas where the gray value of the image changes greatly. However, the edges of objects in the music signal are small enough to be clear, and the overall image looks excessively smooth.

The signal-to-noise ratio and subjective perception determine the quality of a music signal. During the experiment, we compared the signal-to-noise ratio of the music signal before and after processing and extracted the edge of the music signal with the same method to judge the influence of denoising on the edge information. Figure 7 shows a ladder diagram of the filter resolution of the music signal. It can be seen that the number of iterations required for the P-M model to achieve the best PSNR output music signal has reached 147 . The output music signal is relatively fuzzy and the best PSNR is only $17.141 \mathrm{~dB}$, and the MSE is 0.941, which is higher. For the P-M model, if the size of the input music signal is about partial differential equation, it generally requires about 110 iterations, and the best output PSNR does not exceed $18 \mathrm{~dB}$. The number of iterations required for the forward/backward anisotropic diffusion model is greatly reduced to 49 (the input of partial differential equation size is generally less than 60 times), the edge clarity of the output music signal is improved, but a lot of noise is left. There are still chromatic aberrations in each area, which results in the final PSNR which is still only $18.119 \mathrm{~dB}$. 


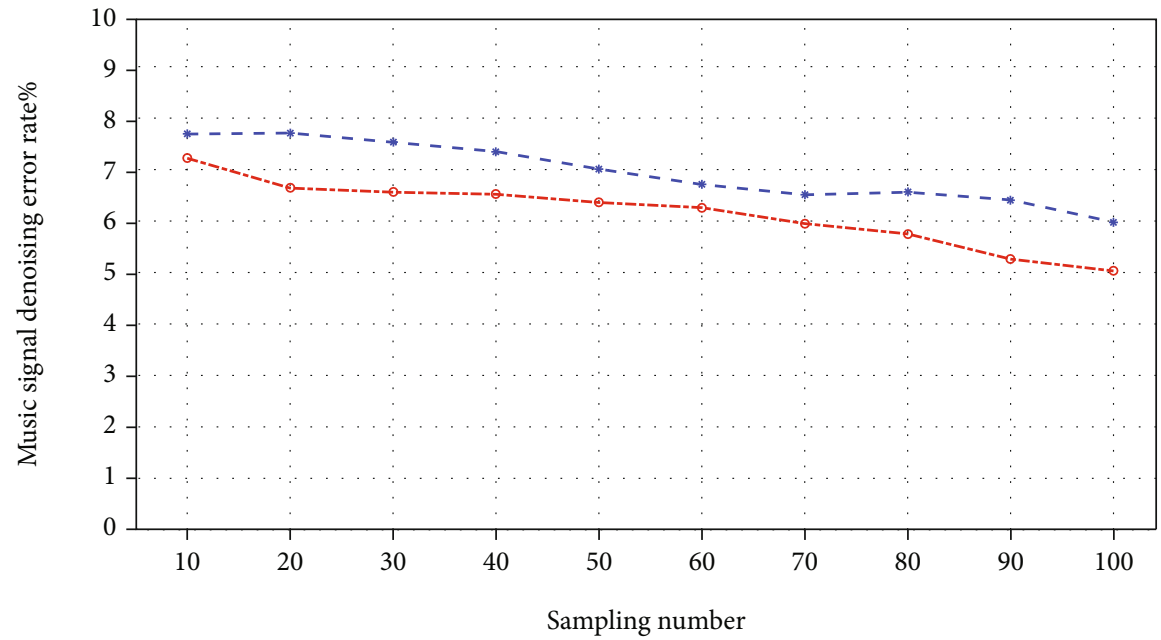

$-\Theta-$ Group 1

$-*-$ Group 2

Figure 6: Line chart of comparison of music signal denoising error rate.

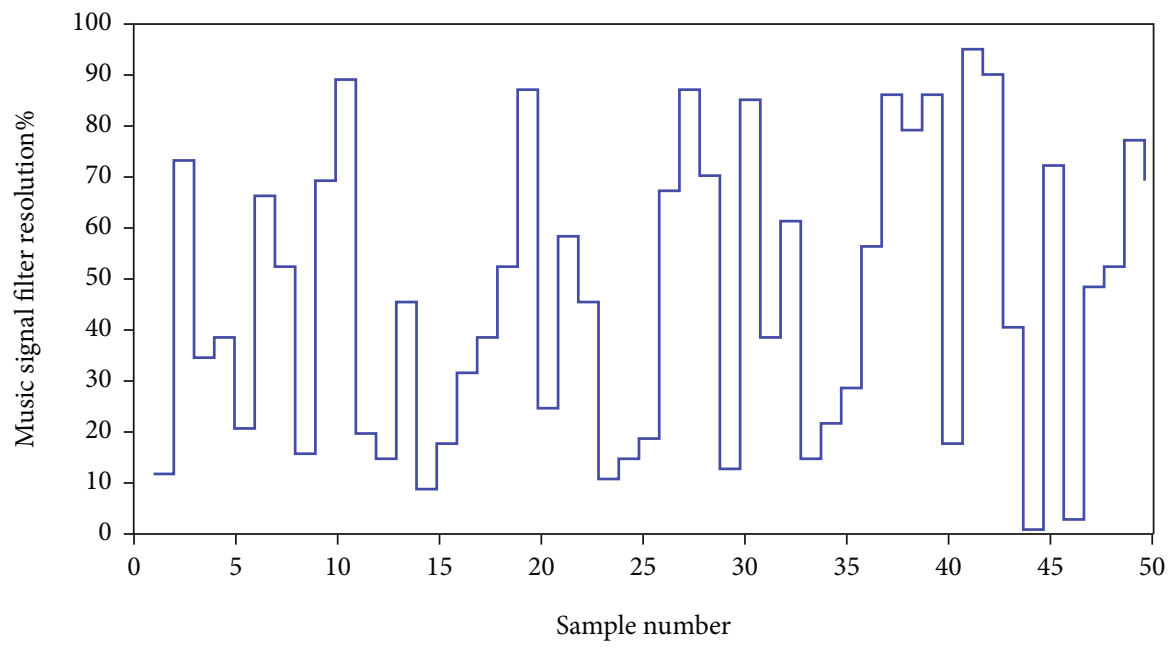

Figure 7: The filter resolution ladder diagram of the music signal.

The overall output of the P-M model is too blurry, and the edges are not clear enough. The best PSNR is only $17.47 \mathrm{~dB}$, and the MSE is high, 0.9176 . The output noise of the forward/backward diffusion model is more, but because of the backward diffusion component, the music signal is not too blurred, and the best output PSNR does not exceed $20 \mathrm{~dB}$. The overall situation of partial differential equation output has been improved, but there is a little error in the music signal, such as dark spots on the larger white area in the main part of the music signal, and the best output PSNR still does not exceed $20 \mathrm{~dB}$. The output of the proposed partial differential equation model suppresses noise very well, and the edges of many small areas can be kept clear without leaving obvious noise. This algorithm effectively improves the results after denoising and improves the peak signal-tonoise ratio. Theoretically speaking, the noise is mainly concentrated in the high-frequency part. In order to combine the advantages between the two and eliminate their short- comings, this paper proposes a denoising method combining partial differential equations and wavelet closed values. The best output PSNR of the partial differential equation model is $22.74 \mathrm{~dB}$, which is significantly improved compared with other algorithms, while the MSE is reduced to 0.306 .

\section{Conclusion}

Based on the theory of partial differential equation method, this paper proposes a model of music signal recognition, uses the finite difference method to discretize the continuous model, and discusses the influence of parameter changes on the noise reduction effect in the new model. The original music signal recognition algorithm based on forward and backward diffusion has been improved so that the processed image has better denoising and enhancement effects. Then, according to the characteristics of the inherent mode function music signal and the residual function music signal 
decomposed by the EMD music signal, different stochastic differential filtering strategies are adopted to filter each layer, and the underwater music signal is simulated. Finally, the inverse group shift Fourier transform is used to obtain the time-dependent solution of the probability density function of the noise FM interference signal after passing the pulse intermediate frequency filter. The results show that the method proposed in this paper has a good suppression effect on nonlinear noise interference. The experimental results show that this model has the enhanced edge characteristics of the antidiffusion algorithm; the addition of numerical statistical filtering not only eliminates the instability of the antidiffusion but also removes the impulse noise in the music signal well and has a good enhancement edge and noise elimination ability. This paper proposes a new music signal denoising algorithm based on the principle of threshold denoising, which performs initial denoising on the original music signal. On this basis, the relationship of the partial differential equation in the denoising process of music signals was studied, a new threshold denoising method was obtained, and a higher signal-to-noise ratio was obtained. Numerical test results show that by using this method to denoise the music signal, the purpose of protecting edges and removing noise is achieved. The use of partial differential equations for music signal processing not only puts forward new research topics for the theoretical research of partial differential equations but also plays an important role in promoting the development of music signal processing, signal analysis, and other issues, so it has important theoretical value and has broad application prospects.

\section{Data Availability}

The data used to support the findings of this study are available from the corresponding author upon request.

\section{Conflicts of Interest}

The author declares that there are no known competing financial interests or personal relationships that could have appeared to influence the work reported in this paper.

\section{References}

[1] T. Yanan, L. Hongwei, and L. Jinghong, "A blind guidance system based on GCADSF image enhancement and music display," in 2017 29th Chinese Control And Decision Conference (CCDC), pp. 4864-4867, Chongqing, 2017.

[2] L. Calkins, R. Khodayi-mehr, W. Aquino, and M. M. Zavlanos, "Sensor planning for model-based acoustic source identification," in 2020 American Control Conference (ACC),, pp. 2679-2684, Denver, CO, USA, 2020.

[3] H. Zuo, Z. Yang, C. Xu, S. Tian, and X. Chen, "Damage identification for plate-like structures using ultrasonic guided wave based on improved MUSIC method," Composite Structures, vol. 203, pp. 164-171, 2018.

[4] V. Lostanlen, J. Andén, and M. Lagrange, "Extended playing techniques: the next milestone in musical instrument recogni- tion," Digital Libraries for Musicology, vol. 2018, pp. 8-10, 2018.

[5] M. Zhang, M. Li, J. Zhang, L. Liu, and H. Li, "Onset detection of ultrasonic signals for the testing of concrete foundation piles by coupled continuous wavelet transform and machine learning algorithms," Advanced Engineering Informatics, vol. 43, article 101034, 2020.

[6] B. Stasiak, J. Mońko, and A. Niewiadomski, "Note onset detection in musical signals via neural-network-based multi-ODF fusion," International Journal of Applied Mathematics and Computer Science, vol. 26, no. 1, pp. 203-213, 2016.

[7] G. Li and B. Zhang, "A novel weak signal detection method via chaotic synchronization using Chua's circuit," IEEE Transactions on Industrial Electronics, vol. 64, no. 3, pp. 2255-2265, 2017.

[8] Y. L. Hsu, J. S. Wang, W. C. Chiang, and C. H. Hung, "Automatic ECG-based emotion recognition in music listening," IEEE Transactions on Affective Computing, vol. 11, no. 1, pp. 85-99, 2020.

[9] F. Zhang, H. Meng, and M. Li, "Emotion extraction and recognition from music," in 2016 12th International Conference on Natural Computation, Fuzzy Systems and Knowledge Discovery (ICNC-FSKD), pp. 1728-1733, Changsha, China, 2016.

[10] L. Chen, Y. S. Choy, K. C. Tam, and C. W. Fei, "Hybrid microphone array signal processing approach for faulty wheel identification and ground impedance estimation in wheel/rail system," Applied Acoustics, vol. 172, article 107633, 2021.

[11] M. Farrokhmanesh and A. Hamzeh, "Music classification as a new approach for malware detection," Journal of Computer Virology and Hacking Techniques, vol. 15, no. 2, pp. 77-96, 2019.

[12] M. Todisco, H. Delgado, and N. W. D. Evans, "A new feature for automatic speaker verification anti-spoofing: constant $Q$ cepstral coefficients," Odyssey, vol. 2016, pp. 283-290, 2016.

[13] H. Shahabi and S. Moghimi, "Toward automatic detection of brain responses to emotional music through analysis of EEG effective connectivity," Computers in Human Behavior, vol. 58, pp. 231-239, 2016.

[14] S. Ando, "Exact FFT-based identification of autoregressive (AR) model," The Journal of the Acoustical Society of America, vol. 146, no. 4, pp. 2846-2846, 2019.

[15] Y. Li and W. Zheng, "Emotion recognition and regulation based on stacked sparse auto-encoder network and personalized reconfigurable music," Mathematics, vol. 9, no. 6, 2021.

[16] R. Sarno, J. A. Ridoean, D. Sunaryono, and D. R. Wijaya, "Classification of music mood using MPEG-7 audio features and SVM with confidence interval," International Journal on Artificial Intelligence Tools, vol. 27, no. 5, article 1850016, 2018.

[17] S. Orcioni, A. Terenzi, S. Cecchi, F. Piazza, and A. Carini, "Identification of Volterra models of tube audio devices using multiple-variance method," Journal of the Audio Engineering Society, vol. 66, no. 10, pp. 823-838, 2018.

[18] G. Piccinni, F. Torelli, and G. Avitabile, "Innovative DOA estimation algorithm based on Lyapunov theory," IEEE Transactions on Circuits and Systems II: Express Briefs, vol. 67, no. 10, pp. 2219-2223, 2020.

[19] X. Jiang, S. Mahadevan, and Y. Yuan, "Fuzzy stochastic neural network model for structural system identification," Mechanical Systems and Signal Processing, vol. 82, pp. 394-411, 2017. 
[20] L. Shu, J. Xie, M. Yang et al., "A review of emotion recognition using physiological signals," Sensors, vol. 18, no. 7, 2018.

[21] G. Li, M. Rong, X. Wang, X. Li, and Y. Li, "Partial discharge patterns recognition with deep convolutional neural networks," in 2016 International Conference on Condition Monitoring and Diagnosis (CMD), pp. 324-327, Xi'an, China, 2016. 